\title{
artigo
}

\section{Validação do conteúdo do instrumento de triagem e classificação de risco utilizado nas unidades de pronto atendimento}

\author{
Validation of the content of the risk classification and screening instrument used in emergency units \\ Validación del contenido del instrumento de triaje y clasificación de riesgo utilizado en las unidades de urgencias
}

\begin{abstract}
RESUMO
Objetivo: validar a parte do conteúdo da Pré Consulta do instrumento utilizado na Triagem e Classificação de Risco da Unidade de Pronto Atendimento de João Pessoa -PB. Método: pesquisa metodológica, de abordagem quantitativa, do tipo validação do conteúdo, realizada com 24 enfermeiros especialistas em urgência em emergência. A análise foi realizada em um software estatístico de forma descritiva por meio de frequências relativas e absolutas. Resultados: foram elencadas facetas para o julgamento do conteúdo do instrumento, a saber: Clareza/linguagem, Disposição do instrumento, Classificação de Risco, Julgamento clínico, Comunicação e fluxo organizacional. A média no tocante às facetas apresentou-se baixo do ponto de vista dos juízes. Através das sugestões e comentários dos especialistas. Conclusão: diante dos resultados alcançados, o referido checklist de Classificação de Risco possui lacunas quanto à clareza, linguagem, organização dos itens, julgamento clínico e comunicação. A partir disso, foi elaborada uma nova proposta de instrumento.
\end{abstract}

DESCRITORES: Enfermagem em Emergência; Assistência Pré-Hospitalar; Triagem.

\section{ABSTRACT}

Objective: to validate the part of the content of the Pre-Consultation of the instrument used in the Screening and Risk Classification of the Emergency Unit of João Pessoa -PB. Method: methodological research, with a quantitative approach, of the content validation type, carried out with 24 specialist nurses in emergency urgency. The analysis was performed in a statistical software in a descriptive way by means of relative and absolute frequencies. Results: facets were listed to judge the content of the instrument, namely: Clarity/language, Instrument layout, Risk Classification, Clinical judgment, Communication and organizational flow. The average in terms of facets was low from the judges' point of view. Through the suggestions and comments of the experts. Conclusion: in view of the results achieved, the aforementioned Risk Classification checklist has gaps in terms of clarity, language, organization of items, clinical judgment and communication. Based on this, a new instrument proposal was elaborated.

DESCRIPTORS: Emergency Nursing; Prehospital Care; Triage.

\section{RESUMEN}

Objetivo: validar la parte del contenido de la Pre-Consulta del instrumento utilizado en el Cribado y Clasificación de Riesgos de la Unidad de Emergencia de João Pessoa -PB. Método: investigación metodológica, con abordaje cuantitativo, del tipo de validación de contenido, realizada con 24 enfermeras especializadas en urgencia de emergencia. El análisis se realizó en un software estadístico de forma descriptiva mediante frecuencias relativas y absolutas. Resultados: se enumeraron las facetas para juzgar el contenido del instrumento, a saber: claridad / lenguaje, disposición del instrumento, clasificación de riesgos, juicio clínico, comunicación y flujo organizacional. El promedio en términos de facetas fue bajo desde el punto de vista de los jueces. A través de las sugerencias y comentarios de los expertos. Conclusión: a la vista de los resultados obtenidos, la lista de verificación de Clasificación de Riesgos antes mencionada presenta lagunas en cuanto a claridad, lenguaje, organización de ítems, juicio clínico y comunicación. En base a esto, se elaboró una nueva propuesta de instrumento.

DESCRIPTORES: Enfermería de Urgencia; Atención Prehospitalaria; Triaje.

RECEBIDO EM: 23/01/2021 APROVADO EM: 02/03/2021 
Deborah Helena Batista Leite

Graduanda em Enfermagem. Universidade Federal da Paraíba. Centro de Ciências da Saúde, João Pessoa, PB, Brasil. ORCID: 0000-0002-9745-9998

\section{Sônia Maria Josino dos Santos}

Doutora em Enfermagem. Universidade Federal da Paraíba. Centro de Ciências da Saúde, João Pessoa, PB, Brasil. ORCID: 0000-0002 8368-1301

\section{Ana Caroline Lima do Nascimento}

Graduanda em Enfermagem. Universidade Federal da Paraíba. Centro de Ciências da Saúde, João Pessoa, PB, Brasil. ORCID: 0000-0002-8631-3715

\section{Gleydson Henrique de Oliveira Dantas}

Enfermeiro. Universidade Federal da Paraíba. Centro de Ciências da Saúde, João Pessoa, PB, Brasil.

ORCID: 0000-0002-0690-5180

\section{Hannah Karolyne Vieira de Lucena}

Residente em Enfermagem. Instituto de Medicina Integral Professor Fernando Figueira. Recife, PE, Brasil. ORCID: 0000-0002-0821-3655

\section{Ismênia Maria Lucena de Medeiros}

Enfermeira. Faculdade Integradas de Patos. Patos, PB, Brasil.

ORCID: 0000-0002-3915-1004

\section{INTRODUÇÃO}

0 $s$ serviços de urgência e emergência são um importante elemento na rede de assistência à saúde no Brasil. Em virtude da alta demanda de procura apresentam dificuldade, o que geram problemas organizacionais, como o atendimento por ordem de chegada, sem estabelecimento de critérios clínicos e superlotação do serviço, podendo acarretar graves prejuízos aos pacientes ${ }^{1}$.

No Brasil um dos componentes da Rede de Atenção às Urgências (RAU) éo Atendimento pré-hospitalar fixo - Unidades de Pronto Atendimento (UPAs), sendo um serviço que atende diariamente um grande número de pacientes que buscam a urgência e emergência por vários motivos, dentre eles por ser "porta-aberta", com possibilidade de oferecer pronta resposta 2 .

Como estratégia frente aos desafios relacionados à organização dos serviços de urgência, o Acolhimento com Classificação de Risco foi apontado como dispositivo de mudança no trabalho da atenção, gestão e produção na saúde ${ }^{3}$.

O enfermeiro éo profissional que responde pela classificação de risco, munido de experiência em serviço de urgência e emergência, através da Resolução n²3/2012 do COFEN que confere privativamente ao enfermeiro, a função de atuar na classificação de risco, exigindo competência técnica e científica em sua execução.

Sabendo que os instrumentos utilizados para embasar e guiar o enfermeiro na Triagem e Classificação de Risco são ferramentas essenciais para o aprimoramento da prática clínica e operacionalização do cuidado, o instrumento utilizado nas UPAs pelos enfermeiros deve ser capaz de proporcionar humanização do atendimento, acesso, resposta rápida e satisfatória ao usuário em condições críticas de saúde e, para tanto, necessita ser submetido a um processo de validação por experts, para que estes profissionais possam opinar em relação à adequação do instrumento ${ }^{4}$.

Diante desta realidade, é possível validar um instrumento com embasamento científico para padronizar e orientar a consulta de enfermagem no contexto do Acolhimento com Classificação de Risco e Triagem?

A pré consulta de enfermagem no contexto do Acolhimento, Classificação de Risco e Triagem deve ser capaz de garantir o planejamento da assistência efetiva e suficiente para promover a continuidade do cuidado ao paciente.

A partir dos pressupostos teóricos que sustentam a pesquisa, esse estudo persegue a hipótese de que o instrumento utilizado na UPA não é suficiente para atender de forma sistema- tizada e efetiva de acordo com a perspectiva da Classificação de Risco e Triagem.

Diante da necessidade da garantia para o cuidado de qualidade e isenção de riscos ao paciente, bem como o reconhecimento da multiplicidade de fatores que cercam o processo da Classificação de Risco e, aliada ao conceito de que a continuidade da assistência se fundamenta no histórico anterior e, portanto, na classificação e pré consulta, justifica-se a importância de realização do presente estudo que tem por objetivo validar a parte do conteúdo da Pré Consulta do instrumento utilizado na Triagem e Classificação de Risco da Unidade de Pronto Atendimento de João Pessoa -PB.

\section{MÉTODO}

Pesquisa metodológica, quantitativa, do tipo validação do conteúdo da pré consulta do instrumento utilizado na Triagem e Classificação de risco - Ficha de atendimento da UPA de João Pessoa PB, pela opinião de especialistas. A validação foi realizada no entre os meses de agosto e setembro de 2020.

A primeira etapa da pesquisa se deu pela busca de possíveis especialistas aptos para avaliação do instrumento. Nesse ínterim, no passo seguinte os especialistas foram definidos. Neste aspecto, não houve consenso sobre quem 


\section{artigo}

poderia ser considerado um especialista, mas alguns critérios foram observados na escolha. Portanto, selecionar enfermeiros para avaliar a pertinência ou relevância do conteúdo, ou seja, definir quem são os enfermeiros especialistas, constituiu-se em um desafio. $\mathrm{O}$ amplo conhecimento dos especialistas na área objeto da validação deve uma premissa a ser considerada.

No que diz respeito aos critérios de seleção de especialistas e a utilização do Coeficiente de Validade de Conteúdo (CVC), foram utilizadas as recomendações propostas por Fehring', o qual recomenda uma amostra de 25 a 50 especialistas. Para tanto, foram considerados como critérios elegíveis para compor a amostra de especialistas aptos a validarem o instrumento, àqueles que possuem experiência na prática clínica na área de urgência e emergência, além de produção científica na temática de estudo.

O tamanho da amostra de especialistas foi estabelecido com base numa proporção de 85\% de ESPECIALISTAS QUE CONSIDERARAM PERTINENTES cada componente avaliado. Admitiu-se uma diferença de 15\% nessa PROPORÇÃO, incluindo um intervalo de 70 a $100 \%$ na referida PROPORÇÃO. Desse modo, para determinar o tamanho da amostra adotou-se uma fórmula a qual considera a proporção final de sujeitos em relação a uma determinada variável dicotômica e a diferença máxima aceitável dessa proporção.

A caracterização da amostra foi calculada pelo Statistical Package for the Social Sciences (SPSS) versão 20.1 de forma descritiva, por frequências relativas e absolutas, com as variáveis: sexo, idade, estado de residência, maior titulação, área de especialização doutorado (quando aplicável), exercício profissional, área do mestrado e/ou doutorado (quando aplicável) e participou ou ministrou curso e/ou disciplina relacionada a classificação de risco.

O cálculo do CVC para o item de pré-consulta do instrumento foi realizado de acordo com a fórmula recomendada por Pasquali' ${ }^{6}$ Foi realizado ainda o cálculo do erro Pei por meio de fórmula, resultando no cálculo do CVC final e adotado o percentual de concordância de $80 \%(0,80)$ para indicação de pertinência dos itens ${ }^{7}$.

Dos 31 especialistas convidados, 24 aceitaram participar do estudo, para os quais foi enviado por e-mail a Carta convite, Termo de Consentimento Livre e Esclarecido e garantido o direito ao anonimato bem como a oportunidade de recusar a participação na pesquisa ou dela retirar-se, quando desejasse, e explicados as etapas do estudo, exposição dos objetivos e do método da pesquisa e, por meio de um formulário do Google foi enviado o instrumento para a avaliação/validação pelos especialistas dos itens constantes na parte da "pré-consulta" do checklist utilizado para Triagem e Classificação de Risco utilizado nas UPAs de João Pessoa - PB. Os especialistas responderam em uma escala de likert variando entre: concordo totalmente, concordo, não concordo nem discordo, discordo e discordo totalmente. A afirmativas foram categorizadas em facetas e o cálculo da média foi feito de acordo com a quantidade de afirmativas referentes a ele para posterior cálculo do CVC.

O projeto foi aprovado pelo Comitê de Ética e Pesquisa do CCS/UFPB, cumprindo os critérios estabelecidos pela Resolução n466/2012 do Conselho Nacional de Saúde, que regulamenta as pesquisas envolvendo seres humanos. A anuência para execução da pesquisa foi obtida sob o número 2.674.218.

\section{RESULTADOS}

Foi possível observar que $58,3 \%$ dos especialistas eram do sexo feminino, tinham idade maior que 30 anos e eram residentes do estado da Paraíba $(\mathrm{n}=14)$. Além disso, a maioria dos especialistas atuavam na área hospitalar (11; 45,8\%), tinham especialização (11; 45,8\%), sendo predominante a especialização em urgência e emergência (12; 49,9\%). Constatou-se então que os especialistas participantes do estudo possuíam conhecimento teórico-técnico, bem como experiência, vivência profissional e acadêmica no que tange às questões norteadoras das temáticas de urgência e emergência. Este dado confere ao grupo de enfermeiros competência para atuarem como especialistas.

As respostas dos especialistas para o item "pré-consulta" do checklist de triagem e classificação de risco variaram numa pontuação de 0 a 5 de acordo com facetas clareza/linguagem, disposição do instrumento, classificação de risco, julgamento clínico, comunicação e fluxo.

A média do item no tocante às facetas apresentou-se baixo do ponto de vista dos juízes, a saber: 0,37 entre a comunicação de sentimentos expressos pelo paciente, 0,41 para julgamento clínico, 0,42 na disposição do instrumento, 0,46 no fluxo organizacional e 0,47 na clareza e linguagem e classificação do risco do paciente.

Em relação ao item Clareza/linguagem, uma especialista sugeriu que alguns termos do checklist fossem ajustados, a exemplo: trocar o termo "chocado" por "sinais de choque", uma vez que se trata da validação de um instrumento legal que irá compor o prontuário do paciente. Outro especialista, sugeriu incluir a pontuação do índice de choque, "por ser de grande valor prognóstico e de fácil aplicação". Em virtude da coerência das sugestões dos especialistas, estas sugestões foram acatadas e modificadas no instrumento.

Uma especialista sugeriu para que sejam listadas outras condições a exemplo de: dor, presença ou não de lesões (expostas, fechadas) e também sugeriu "Dividir a avaliação em pacientes clínicos e traumáticos". Estas sugestões foram aceitas e modificadas no instrumento.

Ainda em relação ao item de avaliação geral do paciente, uma especialista sugeriu que seria interessante "adicionar dados do exame neurológico básico". Outras sugestões dos especialistas a respeito da disposição dos itens do checklist de pré consulta foram atendidas, a saber: "ordenar por sistemas e níveis de gravidade, detalhando mais a questão da pré consulta com as queixas do paciente". Bem como separar por gravidade e dispor de espaço para classificar de acordo com o "protocolo de triagem e classificação de risco (como o de Manchester)".

A tabela 1 demonstra o CVC intravaliador e o CVC geral do instrumento sendo este classificado como baixo $(0,53)$ comprovando a hipótese alternativa do estudo de que a parte instrumento que contempla o item Pré-consulta do instrumento utilizado para Triagem e Classificação de Risco de pacientes atendidos em UPAs de João Pessoa, é insuficiente para atendimento ao paciente.

Através das sugestóes e comentários dos especialistas foi elaborada uma proposta de instrumento pós validação de conteúdo. A proposta visa um instrumento prático e objetivo, com intuito de sistematizar a classificação de risco e acolhimento de acordo com o julgamento clínico do profissional enfermeiro. 


\section{DISCUSSÃO}

Observou-se a predominância de especialistas do sexo feminino, corroborando com outros estudo ${ }^{8,9}$. Isso se explica pelo fato de que na enfermagem ainda prevalece o gênero feminino, evidenciando a caracterização da atuação de mulheres nesta categoria profissional ${ }^{10}$.

Os resultados da pesquisa reforçam que os especialistas da amostra têm perfil ideal no que diz respeito aos critérios para participarem, haja vista a qualificação dos mesmos na temática do estudo. É necessário, além da prática, aprofundamento científico no campo específico para favorecer o raciocínio lógico e aumentar a experiência ${ }^{11}$.
$\mathrm{Na}$ classificação de risco, a investigação deve ser direcionada à principal queixa ou incidente que levou o paciente a procurar atendimento e, esta queixa deve ser bem investigada, visto que uma melhor investigação da queixa principal, eleva o nível de prioridade atribuído ao paciente ${ }^{12}$. Emerge desse contexto, a necessidade de avaliação crítica com maior atenção e rigor da utilidade clínica, ou seja, é necessário que os itens dispostos sejam capazes de mensurar e ter maior aplicabilidade no contexto de pré- consulta.

A classificação de risco e acolhimento são estratégias de atendimento que propõem ao enfermeiro agilidade na assistência às urgências, garantindo prioridade de atendimento para os casos mais graves ou com maior risco. Para tan-

Tabela - Coeficiente de validade de conteúdo intravaliador e geral do item "pré-consulta" do checklist. João Pessoa, Paraíba, Brasil. 2020 (N=24)

\begin{tabular}{|c|c|c|}
\hline JUIZ & MÉDIA & CVC INTRAVALIADOR \\
\hline 1 & 2,66 & 0,53 \\
\hline 2 & 2,10 & 0,42 \\
\hline 3 & 4,29 & 0,86 \\
\hline 4 & 2,25 & 0,45 \\
\hline 5 & 2,94 & 0,59 \\
\hline 6 & 3,67 & 0,73 \\
\hline 7 & 2,41 & 0,48 \\
\hline 8 & 3,24 & 0,65 \\
\hline 9 & 2,54 & 0,51 \\
\hline 10 & 2,16 & 0,43 \\
\hline 11 & 4,69 & 0,94 \\
\hline 12 & 3,79 & 0,76 \\
\hline 13 & 2,30 & 0,46 \\
\hline 14 & 3,35 & 0,67 \\
\hline 15 & 3,67 & 0,73 \\
\hline 16 & 1,55 & 0,31 \\
\hline 17 & 2,98 & 0,60 \\
\hline 18 & 2,48 & 0,50 \\
\hline 19 & 1,48 & 0,30 \\
\hline 20 & 1,98 & 0,40 \\
\hline 21 & 1,98 & 0,40 \\
\hline 22 & 1,83 & 0,37 \\
\hline 23 & 2,00 & 0,40 \\
\hline 24 & 1,75 & 0,35 \\
\hline CVC & & 0,53 \\
\hline
\end{tabular}

to, estar de posse de um instrumento direcionado e organizado com itens que listem as prioridades baseadas na dinâmica do serviço, facilita o atendimento e o processo de trabalho ${ }^{13}$.

Um estudo sobre a importância da avaliação neurológica na classificação de risco, afirma que as patologias neurológicas são altamente prevalentes em Unidades de Pronto Atendimento e envolvem situações extremamente graves em que a atuação rápida e efetiva impacta diretamente no prognóstico do paciente ${ }^{14}$. Reconhecendo a importância da sugestão para melhorar a avaliação neurológica do paciente, os dados para avaliar esse domínio foram incluídos no instrumento.

As sugestões a respeito da disposição dos itens do checklist de pré consulta corroboram com o protocolo de Classificação de Risco e Triagem de Manchester, no qual o Protocolo deve ser baseado em níveis de prioridade clínica, avaliados mediante os sinais e sintomas relatados pelo paciente ${ }^{15}$.

Classificar pacientes quanto à gravidade clínica é inerente a prática de enfermeiros emergencistas. Isto posto, a tomada de decisão do enfermeiro deverá ser criteriosa e baseada na identificação do problema, por meio da coleta de dados efetiva e direcionada, com intuito de identificar a queixa principal para então realizar a classificação de risco adequadamente ${ }^{16}$.

Entretanto, para que se consiga avaliar o estado geral do paciente, identificar os fatores associados ao quadro clínico e classificar a prioridade de atendimento, o enfermeiro necessita que o instrumento utilizado seja organizado em fluxos coesos e bem delimitados que garantam a continuidade do atendimento no serviço de urgência ${ }^{17}$.

$\mathrm{O}$ uso de checklist previne erros e falhas humanas, através de método sistematizado e mediante controles e padrões de segurança. Destarte, as informações armazenadas no instrumento podem servir para fornecer respaldo legal para a instituição e profissionais ${ }^{18}$.

\section{CONCLUSÃO}

Diante dos resultados alcançados, comprova-se que o referido checklist de Classificação de Risco possui lacunas quanto à 


\section{artigo}

Leite, D.H.B.; Santos, S.M.J.; Nascimento, A.C.L.; Dantas, G.H.O.; Lucena, H.K.V.; Medeiros, I.M.L.;

Validação do conteúdo do instrumento de triagem e classificação de risco utilizado nas unidades de pronto atendimento

clareza, linguagem, organização dos itens, julgamento clínico e comunicação. Inferindo-se portanto, que o conteúdo do instrumento que é utilizado na UPA não é suficiente para atender aos pressupostos que sustentam perspectiva a Classificação de Risco e Triagem. Os resultados do presente estudo confirmam a hipótese estabelecida do trabalho.

Após a análise e discussão dos resulta- dos, houve a necessidade de elaborar uma proposta de intervenção com adequação do conteúdo do instrumento de pré consulta, que foi construída através das sugestôes dos especialistas e da realidade dos serviços de pronto atendimento.

Ademais, o presente estudo contribuirá de forma substancial para suscitar a atenção dos profissionais quanto à importância das ade- quações de um instrumento de pré consulta que possibilite um cuidado seguro no serviço de emergência, de modo a cooperar para um melhor prognóstico para o paciente.

Logo, recomenda-se que outras pesquisas sejam realizadas para a construção e validação de instrumentos voltados para os âmbitos da emergência, avaliando também o cuidado multiprofissional. -

\section{REFERÊNCIAS}

1. Souza CC, Araújo FA, Chianca TCM. Scientific literature on the reliability and validity of the manchester triage system (mts) protocol: a integrative literature review. Rev Esc Enferm USP. [Internet]. 2015;49(1):144-51. Available from: https://doi. org/10.1590/S0080-623420150000100019

2. Cassettari SSR, Mello ALSF. Demand and type of care provided in emergency services in the city of Florianópolis, Brazil. Texto \& Contexto Enferm. [Internet]. 2017;26(1):e3400015. Available from: https://doi.org/10.1590/0104-07072017003400015

3. Rodrigues CK, Malderran R, Novo NF. Acolhimento com classificação de risco em um pronto socorro do município de São Paulo: análise dos desfechos dos casos. Rev Recien [Internet]. 2019;9(28):137-45. Available from: https://www.recien.com.br/ index.php/Recien/article/view/325/pdf

4. Santos SMJ. Dor aguda no infarto agudo do miocárdio. Revisão do Diagnóstico de Enfermagem. 1st ed. Novas Edições Acadêmicas; 2017. 194 p.

5. Fehring RJ. The Fehring model. In: Carroll-Johnson P. Classification of nursing diagnosis: proceedings of the tenth conference of North American Nursing Diagnoses Association. Philadelphia: Lippincott; 1994. p. 59.

6. Pasquali L. Psicometria: teoria dos testes na psicologia e na educação. 1st ed. Editora Vozes; 2017. 481 p.

7. Polit DF, Beck CT. The content validity index: Are you sure you know what's being reported? critique and recommendations. Res Nurs Health [Internet]. 2006;29(5):489-97. Available from: http://doi.wiley.com/10.1002/nur.20147

8. Teixeira GS, Silveira RCP, Mininel VA, Moraes JT, Ribeiro IK da S. Quality of life at work and occupational stress of nursing in an emergency care unit. Texto \& Contexto Enferm. [Internet]. 2019;28:e20180298. Available from: http://dx.doi. org/10.1590/1980-265x-tce-2018-0298

9. Feliciano WLL, Lanza LB, Pinto VAB. As representações sociais dos usuários dos serviços de saúde sobre o homem na enfermagem. Rev Fac Cienc Med Sorocaba. [Internet]. 2019;21(1):15-21. Available from: https://doi. org/10.23925/1984-4840.2019v21i1a4

10. Barros MAA, Ferreira PJ de O, Pereira da Silva FM, Holanda R-A, Carneiro SN. Perfil acadêmico do preceptor de enfermagem na atenção primáriaà saúde. Rev Exp Cat Saúde. [Internet]. 2018;2(2):62. Available from: https://dx.doi.org/10.25191/recs.v2i2.2081
11. Oliveira LB. Ensino do pensamento crítico para estudantes de Enfermagem: abordagens, desenho e teoria de uma intervenção educativa [Tese]. São Paulo: Universidade de São Paulo; 2019. Available from: https://www.teses.usp.br/teses/disponiveis/7/7139/tde-09122019-135905/

12. Diniz AS, Silva AP, Souza CC, Chianca TCM. Demanda clínica de uma unidade de pronto atendimento, segundo o protocolo de Manchester. Rev Eletrônica Enferm. [Internet]. 2014;16(2). Available from: https://doi.org/10.5216/ree.v16i2.21700

13. Marques LA, César FCR, Izidoro LCR, Cabral KB, Santos LF, Brasil VV, et al. Satisfação de usuários com o acolhimento e classificação de risco em unidades públicas de saúde. Rev Eletrônica Enferm. [Internet]. 2018;20. Available from: https:// doi.org/10.5216/ree.v20.50113

14. Moraes e Silva M, Schultze ACB, Cavalheiro BP, Meyer LF, Fronchetti JA, Mercer PBS, et al. Profile and generalist physician knowledge about neurology in emergency department: headache management. Arq Neuropsiquiatr. [Internet]. 2020;78(1):44-9. Available from: https:// doi.org/10.1590/0004-282×20190198

15. Paula MIP, Andrade UV. Classificação de risco segundo o protocolo de manchester: uma proposta de humanização nos serviços de urgência e emergência. Rev Mosaicum [Internet]. 2017;13(25):143-58. Available from: https://doi.org/10.26893/ rm.v13i25.98

16. Rates HF, Cavalcante RB, Alves M, Santos RC, Machado RM, Macêdo AS. O (in)visível no cotidiano de trabalho de enfermeiros no acolhimento com classificação de risco. Rev Eletrônica Enferm. [Internet]. 2018;20. Available from: https://doi. org/10.5216/ree.v20.48608

17. Amaya MR, Paixão DPSS, Sarquis LMM, Cruz EDA. Construção e validação de conteúdo de checklist para a segurança do paciente em emergência. Rev Gaúch Enferm. [Internet]. 2016;37(spe):e68778. Available from: https://doi. org/10.1590/1983-1447.2016.esp.68778

18. Girondi JBR, Bussolo P, Rosa LM, Amante LN, Sebold LF, Gelbeck FL, et al. Validação de conteúdo de checklist de intervenções de enfermagem pré-operatórias para angioplastia. Enferm Foco. (Brasília). [Internet]. 2020;11(2):11-7. Available from: http://revista.cofen.gov.br/index.php/enfermagem/article/view/2752/761 